\title{
Central engines for gamma-ray bursts
}

\author{
S.E. Woosley and A.I. MacFadyen
}

Astronomy Department, UCSC, Santa Cruz, CA 95064, U.S.A.

e-mail: woosley@ucolick.org

Received December 29, 1998; accepted March 10, 1999

\begin{abstract}
What powers a gamma-ray burst (GRB)? We discuss here some properties of several currently favored models based on black hole accretion with emphasis on the collapsar - a rotating massive star whose iron core collapse produces a black hole. Depending on mass, rotation rate, and viewing angle, collapsars can explain a wide gambit of GRBs from faint events like GRB 980425, to bright ones like GRB 971214. Because of accretion disk instabilities, the $\Gamma$ in the jet may be rapidly time variable. The burst itself is made by a combination of internal shocks in the jet and external shocks with the pre-explosive stellar wind. Beaming for hard gamma-rays is about $1 \%$, but mildly relativistic matter is ejected at larger angles. All collapsars produce Type Ib/c supernovae like SN 1998bw, but the converse is not true. Most Type $\mathrm{Ib} / \mathrm{c}$ supernovae do not make GRBs.
\end{abstract}

Key words: black holes — accretion; gamma-ray bursts

\section{Introduction}

Modern cosmological models for GRBs are of two varieties: 1) those that attempt to explain the burst itself and its "afterglows", given the prior existence of a highly relativistic jet or fireball having special properties, and 2) models for making that jet. This paper concerns the second kind of model.

Models for the "jet engine" must satisfy a number of constraints. First, the brightest bursts require $\gtrsim 10^{53}$ $f_{\Omega} f_{\gamma}^{-1}$ erg where $f_{\Omega}$ is the beaming factor which, for gamma-rays, is probably not much less than 0.01 (halfopening angle $5-10$ degrees), and $f_{\gamma}$ is the efficiency for converting total jet energy into gamma-rays of $10 \mathrm{keV}$ to a few $\mathrm{MeV}$, perhaps $\sim 0.2$. Second, the jet must have a high $\Gamma$, so that relativistic beaming can keep the duration of the burst short even though the gamma-rays are

Send offprint requests to: S.E. Woosley made in a very large volume. Values of $\Gamma$ of $\gtrsim 100$ are needed to explain most BATSE GRBs. However, as we will discuss later (Sect. 4), smaller $\Gamma \sim 5$ may explain faint events like GRB 980425. If $\Gamma \sim 300$, the corresponding mass is $\lesssim 10^{-4} f_{\Omega} M_{\odot}$. That the requisite mass be this small is referred to as the "baryon loading problem". Finally, the events must occur at a reasonable rate. The exact value depends on the median red shift and is unknown, but $10^{-7} / f_{\Omega}$ per year per $L_{*}$ galaxy is reasonable. At least some fraction of these, perhaps all events lasting long enough to trigger Beppo-Sax ( $\gtrsim 5 \mathrm{~s}$ ), occur in star forming regions. The location of shorter bursts is unknown.

GRBs may also have diverse origins. The association of GRB 980425 with SN 1998bw remains controversial to some, but at $38 \mathrm{Mpc}$, the GRB energy was only about $10^{48} f_{\Omega}$ erg, less than $10^{-5}$ the energy of GRB 971214 . Collapsars have difficulty explaining short (mean duration $0.3 \mathrm{~s}$ ) hard bursts, but merging neutron stars have difficulty explaining long (mean duration $20 \mathrm{~s}$ ), complex ones. And, as has been remarked frequently, the range of GRB light curve shapes is very diverse.

Setting aside for now what one might term "exotic models" - supermassive stars, cosmic strings, physics beyond the standard model, and the like - the requisite conditions described above are best met by a class of models based upon hyperaccreting, stellar mass black holes. These can be realized in several ways (see Popham et al. 1998 for a summary), but have in common a black hole of several solar masses accreting matter from a disk at a rate 0.001 to $10 M_{\odot} \mathrm{s}^{-1}$. The models vary in disk mass, accretion rate, black hole mass and Kerr parameter, and the manner in which accretion energy is channeled into directed relativistic motion, but they have in common that the GRB they produce always signals either the birth or sudden appreciable growth of a black hole. 


\section{MHD energy extraction}

As the black hole accretes, it is spun up, so a large fraction of system's energy comes to reside in its rotation. For neutron star mergers the mass accreted (after black hole formation) may be $\Delta M \sim 0.01-0.1 M_{\odot}$ (Ruffert \& Janka 1998; Eberl 1998); for neutron star - black hole mergers, the mass is $\sim 5$ times greater (Eberl et al. 1998). Thus the total rotational energy in the hole is $\lesssim 10^{54} \mathrm{erg}$. The energy which may be dissipated in the disk is also large, 0.06 to $0.42 \Delta M c^{2} \sim 10^{53} \mathrm{erg}$. However, it is also possible that the matter in the disk accretes without appreciable emission (advection dominated flow).

A number of mechanisms have been discussed for converting the available energy into directed flow: magnetic interaction extracting rotational energy from the hole (e.g., Blandford \& Znajek 1977; Mészáros \& Rees 1997); reconnection of magnetic field amplified by differential rotation in the disk (Thompson 1996; Mészáros \& Rees 1997); the dynamo action of rotating magnetic field in the disk (Katz 1994, 1997); flare-like activity of the disk field (Galeev 1979); etc. These solutions all have in common the need for magnetic field strength near the equipartition value, $\sim 10^{15}$ Gauss. Whether this field is actually created is unclear. The MHD solutions are also appropriate chiefly for low viscosity, low entropy disks that last a long time. It might be hard to generate the necessary magnetic field in a disk that made only a few revolutions. In any case, the lifetime of the disk formed, e.g., by neutron star merger is tens of milliseconds for a disk viscosity, $\alpha \sim 0.01$ (Ruffert \& Janka 1998; Popham et al. 1998). This is much shorter than most GRBs, so the duration would have to be governed by the external interaction, not by the life time of the engine.

There is an interesting conundrum here in that the MHD models need large fields that would imply large disk viscosity (Balbus \& Hawley 1998), but in the merging neutron star case, high viscosity would also cause so much heating that much of the desired energy would be radiated away as neutrinos. On the other hand, the non-MHD models, i.e., those that use neutrinos to transport energy (Ruffert \& Janka 1998), implicitly assume a large disk field in order to provide the necessary viscosity for heating. So MHD energy extraction, if it works at all, should also be important in neutrino powered models, especially in the collapsars of the next section.

Generally speaking, MHD models have several advantages. First, MHD energy extraction can potentially be quite efficient and the production of jets can occur naturally, as in active galactic nuclei. The upper bound on the jet energy is about $10^{53} \mathrm{erg}$ beamed into a small fraction of the sky. If one must confront energetic GRBs at red shift 5 say, this sort of efficiency may be necessary. Second, MHD energy extraction can work for low values of accretion rate (and $\alpha$ ). Below $0.1 M_{\odot} \mathrm{s}^{-1}$, even for high $\alpha$, energy extraction from the disk by neutrino emission

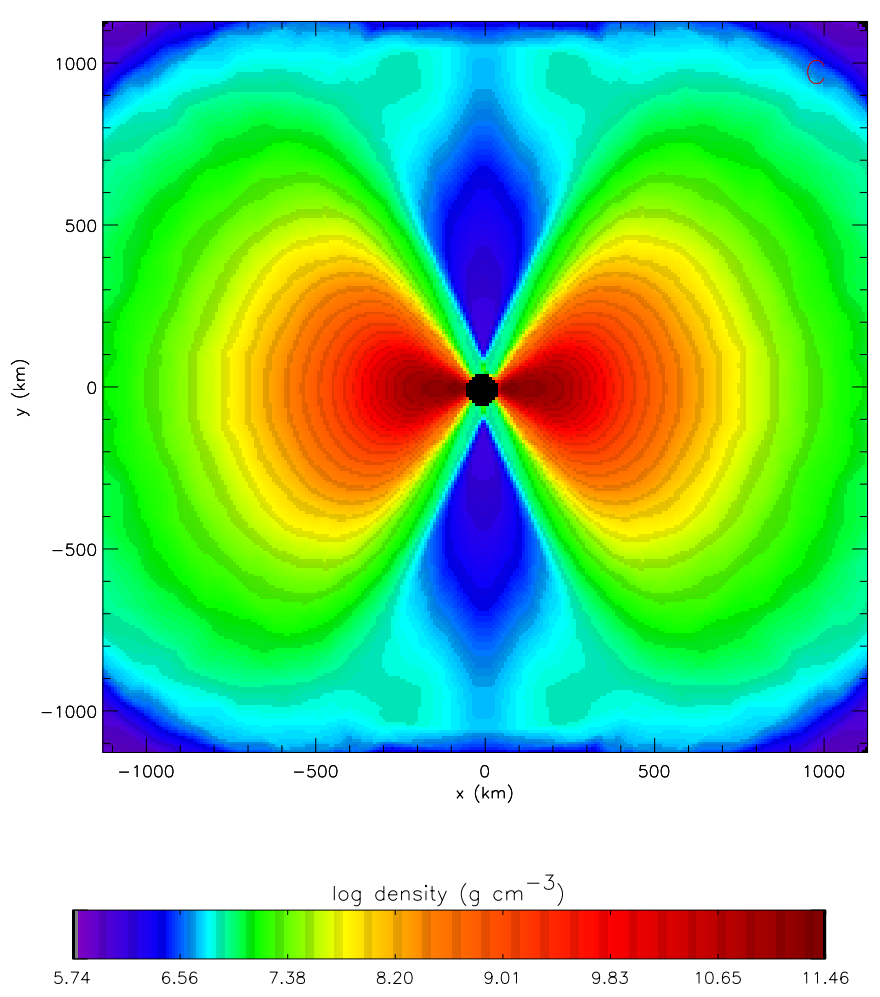

Fig. 1. The disk density structure inside a $14 M_{\odot}$ helium star $7.5 \mathrm{~s}$ after iron core collapse. The polar density is nearly six orders of magnitude lower than the equatorial density near the inner boundary

becomes inefficient (Popham et al. 1998). This is another reason to consider MHD extraction in collapsars.

\section{Collapsars}

Now we consider briefly the collapsar model (Woosley 1993). Because a detailed description of this model has already been accepted for publication (MacFadyen \& Woosley 1998), we will be brief.

A rotating massive star $\left(M \gtrsim 30 M_{\odot}\right)$ reaches the end of its life. Because the iron core is considerably more massive than $2 M_{\odot}$, it is already not far from forming a black hole. Moreover, the density gradient outside that core is shallow, so accretion occurs at a high rate. The core grows and eventually collapses to a black hole which attempts to accrete the rest of the star. However, after about $2-3 \mathrm{~s}$, for $j=5-1510^{16} \mathrm{~cm}^{2} \mathrm{~s}^{-1}$, a centrifugally supported disk forms. Matter falls into the hole along the rotational axis opening up a funnel like density structure (Fig. 1) while matter in the disk accretes more slowly at about $0.1 M_{\odot} \mathrm{s}^{-1}$. Depending upon viscosity and Kerr parameter, (values $\alpha=0.1, a=0.9$ here for example) temperatures in the inner disk reach about $10^{11} \mathrm{~K}$ at a density $\sim 10^{10} \mathrm{~g} \mathrm{~cm}^{-3}$ and radius $10 \mathrm{~km}$.

Over the next $10 \mathrm{~s}$ to $20 \mathrm{~s}$, the black hole grows from $\sim 3 M_{\odot}$ to $5 M_{\odot}$. For the chosen $\alpha$ and calculated accretion 

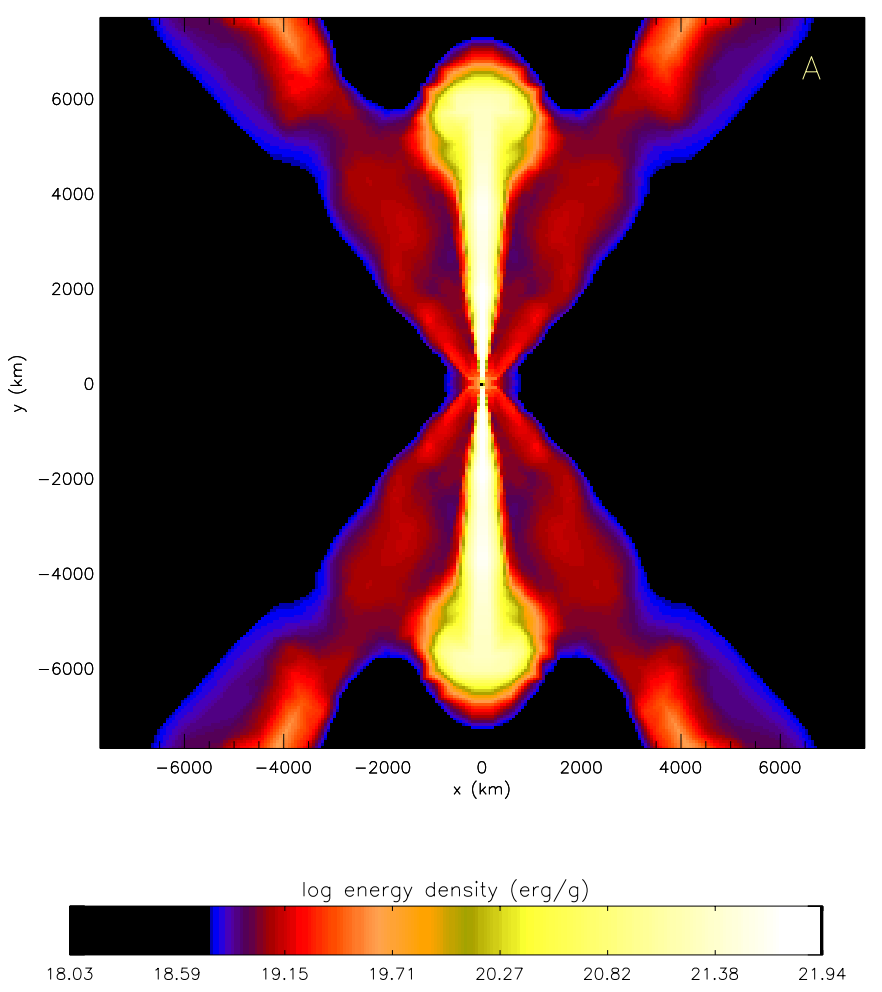

Fig. 2. Depositing $10^{51} \mathrm{erg} \mathrm{s}^{-1}$ along the rotational axis above and below the black hole leads to jet formation, seen here $824 \mathrm{~ms}$ after initiation. The ratio of energy to baryonic rest mass exceeds unity. Were the matter to expand freely it would reach approximately the speed of light

rate, most of the energy dissipated in the disk is radiated as neutrinos, $\sim 1-510^{53} \mathrm{erg}$. A fraction of the neutrinos emitted by the disk encounter their anti-particles coming from the other side of the disk. The large collision angle favors neutrino annihilation and pair production. The electrons and positrons so produced retain the net momentum of the collision which has a component directed outwards along the rotational axis. The geometry of the funnel shaped density profile along the axis, the momentum of the pairs, and the large energy deposition all favor the formation of axial jets, but only after the ram pressure of the infalling matter can be reversed. This takes another few seconds (when the density declines to $10^{5}-10^{6} \mathrm{~g} \mathrm{~cm}^{-3}$ at $50 \mathrm{~km}$ ), The efficiency for neutrino annihilation is not large, typically $1 \%$, so the total energy deposited is in the range $3-2010^{51} \mathrm{erg}$. These numbers are calculated using the disk solutions of Popham et al. (1998) mated smoothly to the 2D hydro calculations of MacFadyen \& Woosley (1998) as described in the latter publication. Neutrino transport is calculated assuming that the disk remains optically thin to neutrinos. This is marginally violated in the inner disk for the largest values of Kerr parameter developed in the problem, hence the uncertainty in the above numbers. More accurate values can be determined in the future.
Whether the energy actually comes predominantly from neutrino annihilation or from MHD processes remains unclear, but from our discussion so far, it makes sense to deposit about $10^{51} \mathrm{erg} \mathrm{s}^{-1}$ near the inner boundary of the rotational axis of the collapsar $(50 \mathrm{~km}$ in MacFadyen \& Woosley) starting about 5-7 s after the iron core collapse and observe the consequences. This was done by MacFadyen \& Woosley and the outcome was bipolar jets with an opening angle of $\lesssim 10$ degrees (Fig. 2). In the non-relativistic code they employed, it was hard to determine the final velocity of the jets (speeds considerably greater than c developed!), but it is clear that the flow will ultimately become relativistic. These calculations have been repeated recently using a special relativistic version of the 2D hydro-code "Prometheus" by Aloy et al. (1999) and velocities approaching c are indeed observed along with very high energy loading factors (up to 100 and more times the baryonic mass in the jet).

One can thus separate the collapsar phenomenon into several stages:

1) the iron core collapses and the disk forms (2s),

2) accretion along the polar column declines to the point where a jet can form and move outwards $(2-5$ more $\mathrm{s})$,

3 ) the jet bores out of the star (radius about $310^{10} \mathrm{~cm}$; about 5 more s),

4) the first interaction of the ejecta with the pre-explosive mass loss of the star makes the first hard X-rays; and finally,

5) the jet continues for about 10 more s fully evacuating the polar column and reaching its asymptotic relativistic speed. Internal shocks in the jet and circumstellar interaction produce the main GRB.

What is seen will also vary with angle. Only at small angles, $\lesssim 10$ degrees, will high $\Gamma$ matter produce a bright GRB. Mildly relativistic matter will be ejected out to a larger angle (subsequent calculations after the meeting show this), approximately 30 degrees. This matter will make a weaker GRB, but actually contains a large fraction of the explosion energy. It will contribute to the afterglow in radio, optical, and X-ray. The jet eventually ends up blowing up the entire star, leaving behind a black hole of $\gtrsim 5 M_{\odot}$.

The total energy in the relativistic jets is (at most) about $10^{52}$ erg in the neutrino powered model, perhaps an order of magnitude more if MHD processes are efficient. The beaming is about $1 \%$. So inferred isotropic energies in bursts of up to $10^{54}$ (neutrino) or $10^{55}$ (MHD) erg can be accommodated (modulo the uncertain efficiency factor for turning jet energy into gamma-rays).

There are several other interesting properties of the collapsar model. First, it will occur in star forming regions in stars that have lost their hydrogen envelope. The jet always blows up the star, so that a supernova of some sort should accompany the GRB is unavoidable. In order to obtain the large helium cores and rotation rates that 
the collapsar model needs, it helps to have less mass loss. So collapsars may preferentially occur in low metallicity star forming regions.

Second, MacFadyen \& Woosley note that the accretion rate into the black hole is not constant in time. Photodisintegration instability may modulate the accretion on a time scale $\sim 50 \mathrm{~ms}$. In the neutrino based collapsar model, and probably also in the MHD one, this rapid variation in accretion rate will give a highly variable $\Gamma$ in the jet, conducive to the formation of internal shocks.

Finally, the wind of a Wolf-Rayet star, even a low metallicity one, is appreciable. The GRB is thus likely to be surrounded by an extended region (perhaps also asymmetrical) of high density. For a mass loss rate of $10^{-5} M_{\odot}$ $\mathrm{y}^{-1}$ and velocity $1000 \mathrm{~km} \mathrm{~s}^{-1}$, a jet of $10^{-4} f_{\Omega} M_{\odot}$ will encounter $1 / \Gamma$ of its mass (for $\Gamma=100$ ) in about $20 \mathrm{AU}$. Variations in mass loss and $\Gamma$ make this an approximate number. Some bursts may be made by internal shocks; some by external shocks as they run into this wind.

\section{SN 1998 bw and GRB 980425}

The equivalence of these two events continues to be debated. We believe they were one and the same; indeed the collapsar model predicts every GRB (long enough to trigger Beppo-Sax) should be accompanied by a Type Ib/c supernovae. That the supernova should be as bright as SN 1998bw (which implies an exceptional quantity of ${ }^{56} \mathrm{Ni}$ ) was not anticipated, but in retrospect is not too surprising. Considerable mass falls into high temperature and is then ejected. It should be clear though that, in SN 1998bw as well as other GRBs, we are dealing with an unusual sort of supernova. Probably $99 \%$ of $\mathrm{SN} \mathrm{Ib} / \mathrm{c}$ are made in $3-4 M_{\odot}$ helium cores that leave neutron stars and make no GRB. Of the remaining $1 \%$, the vast majority beam their GRB in a direction that is not seen. So one should look for supernovae in GRB error boxes, but not expect GRBs in SN Ib/c boxes.

GRB 980425 was faint because the explosion ejected far less energy in the form of relativistic matter than other GRBs. It takes about $10 \mathrm{~s}$ in the collapsar model from iron core collapse until the full development of a very relativistic jet. Moreover, the energy of the jet depends in a very non-linear way on the accretion rate. An accretion rate of $0.05 M_{\odot} \mathrm{s}^{-1}$ produces, in the neutrino model, orders of magnitude less energy than an accretion rate of $0.1 M_{\odot} \mathrm{s}^{-1}$. Perhaps an energetic jet failed to develop or died before $10 \mathrm{~s}$ was up. One would still get a supernova though and high ejection velocities along the rotational axis.

It is also possible that a very energetic jet developed, but, owing to poor collimation properties, was loaded with too much matter to become highly relativistic. Mildly relativistic matter would still be ejected as the jet powered shock wave moved down the density gradient at the surface of the star (McKee \& Colgate 1973). A supernova of $10^{52} \mathrm{erg}$ with its initial explosion focused into $10 \%$ of the sky would eject about $10^{25} \mathrm{~g}$ of matter having $\Gamma=5$ (Woosley et al. 1998) in $10 \%$ of the sky. Running into circumstellar material at a radius of about $510^{12} \mathrm{~cm}$, this would produce a GRB that peaked at about $5 \mathrm{~s}$ $\left(t_{\text {peak }}=2000 E_{48} A_{11}^{-1} \Gamma^{-4} \mathrm{~s}\right.$ for a wind density that fell as $10^{11} A_{11} r_{12}^{-2}$ helium nuclei $\mathrm{cm}^{-3}$; Sari \& Piran 1997a,b; Sari 1998, private communication).

At later times, the shock of the jet passage moves around the star and the explosion becomes more symmetric. There is also a lot more matter and energy moving at $\Gamma \sim 1-2$ that could contribute to X-ray, radio, and optical afterglows. In summary, in order to produce a supernova like SN 1998bw with kinetic energy well in excess of $10^{52}$ erg (Iwamoto et al. 1998; Woosley et al. 1998) powered by black hole formation and asymmetric mass ejection, one expects a GRB very much like 970425 .

Acknowledgements. We are grateful to Alex Heger, John Danziger, Chris Fryer, Jonathon Katz, Bob Popham, and Re'em Sari for valuable discussions of the collapsar model at the meeting and afterwards. This reasearch was supported by the NSF (AST AST-97-31569), NASA (MIT SC A292701), and by the Humboldt Foundation.

\section{References}

Aloy M.A., Müller E., Ibanez J.M., Marti J.M., MacFadyen A., 1999, A\&A (in preparation)

Balbus S.A., Hawley J.F., 1998, Rev. Mod. Phys. 70, 1

Blandford R.D., Znajek R.L., 1977, MNRAS 179, 433

Galeev A.A., Rosner R., Vaiana G.S., 1979, ApJ 229, 318

Iwamoto K., and 26 others, 1998, Nat 395, 672

Katz J., 1994, ApJ 422, 248

Katz J., 1997, ApJ 490, 633

MacFadyen A., Woosley S.E., 1999, ApJ (accepted) astro-ph/9810274

Mészáros P., Rees M.J., 1997, ApJ 482, L29

McKee C.R., Colgate S.A., 1973, ApJ 181, 903

Popham R., Woosley S.E., Fryer C., 1998, ApJ (in press), astro-ph/9807028

Ruffert M., Janka H.-Th., 1998, A\&A (submitted) astro-ph/9809280

Sari R., Piran T., 1997, ApJ 485, 270

Sari R., Piran T., 1997, MNRAS 287, 110

Thompson D.J., 1996, in Gamma-Ray Bursts: 3rd Huntsville Symposium, AIP Conf. Proc 384, Kouveliotou C., Briggs M., Fishman G. (eds.). AIP: New York

Woosley S.E., 1993, ApJ 405, 273

Woosley S.E., Eastman R.G., Schmidt B., 1998, ApJ (in press) astro-ph/9806299 